\title{
SYNTHESIS, CHARACTERIZATION AND IN VITRO ANTIFUNGAL EVALUATION OF TRANSITION METAL COMPLEXES DERIVED FROM $N$ '-SUBSTITUTED-4- METHYLPIPERIDINE-1-CARBOTHIOHYDRAZIDES
}

\author{
Juhi Baranwal ${ }^{1}$, Praveen Kumar ${ }^{2,}$, Manisha Kumari ${ }^{3}$ and V.S. Tiwari ${ }^{4}$ \\ ${ }^{1}$ Department of Chemistry, Ranchi University, Ranchi-834002, Jharkhand, India. \\ ${ }^{2, \bigotimes}$ Department of Pharmaceutical Chemistry, Faculty of Pharmacy, Uttar Pradesh University of \\ Medical Sciences, Saifai, Etawah-206130, Uttar Pradesh, India. \\ ${ }^{3}$ Department of Chemistry, R.T.C. Institute of Technology, Ranchi-835219, Jharkhand, India. \\ ${ }^{4}$ Department of Chemistry, Doranda College, Ranchi-834002, Jharkhand, India. \\ ${ }^{\square}$ Corresponding Author: praveensha77@gmail.com
}

\begin{abstract}
Series of coordination complexes of $\mathrm{Cr}(\mathrm{III}), \mathrm{Ni}(\mathrm{II})$ and $\mathrm{Zn}(\mathrm{II})$ with various $N^{\prime}$-substituted-4-methylpiperidine-1carbothiohydrazides (6a-f) have been synthesized and characterized quantitatively and qualitatively by UV-visible spectroscopy, FTIR spectroscopy, ${ }^{1} \mathrm{H}$ NMR studies; microelemental analysis; electrical conductance values and magnetic susceptibility measurements. The metal complexes displayed greater antifungal activity than corresponding ligands and the order of their activity was found as $[\mathrm{M}(\mathrm{salmpth})(\mathrm{Py})]>\left[\mathrm{M}(\mathrm{fmpth})_{2}\right]>\left[\mathrm{M}(\mathrm{talmpth})_{2}\right]$. The greater antifungal activity of metal complexes was found due to decrease in polarity of metal ions while coordinating with ligands during chelation process. The algacidal effect of few ligands viz. $\mathrm{H}_{2}$ salmpth, Hfmpth and Htalmpth and its chromium(III) complexes were also studied.

Keywords: Synthesis, Transition Metal Complexes, N'-Substituted-4-methylpiperidine-1-carbothiohydrazides, Antifungal Activity, Algacidal Effect. RASĀYAN J. Chem., Vol. 14, No.3, 2021

\section{INTRODUCTION}

Modern coordination chemistry involves various types of strategically designed and functionalized ligand systems. Among them, $N^{\prime}$-substituted-4-methylpiperidine-1-carbothiohydrazides are a salient class of Schiff base ligands in coordination chemistry due to the dual coordinating sites (N and $\mathrm{S}$ ) as potential donors. ${ }^{1-5}$ Moreover, the coordination compounds of transition metal ions with bidentate ligands having $\mathrm{N}$ and $\mathrm{S}$ as donor sites are considered significant due to similarity with redox-active metalloenzymes. ${ }^{6-10}$ Furthermore, the potential of Schiff base ligands has urged the researchers to undertake them as an elite ligand. ${ }^{11,12}$

The synthesis of Schiff base ligands and their corresponding metal complexes in a controlled manner becomes an important strategic pathway. ${ }^{13-16}$ In this study, we chose readily available, low-cost and potential organic molecules as precursors to synthesize the new Schiff base ligands and their metal complexes. The unique advantage of these Schiff base ligands is the change in their ligation behavior depending on the metallic systems and the stoichiometry. ${ }^{17,18}$ Therefore given above said advantages, we had synthesized, characterized the novel Schiff base ligands ( $N^{\prime}$-Substituted-4-methylpiperidine-1carbothiohydrazides, (6a-f) and their corresponding complexes of $\mathrm{Cr}(\mathrm{III}), \mathrm{Ni}$ (II) and $\mathrm{Zn}$ (II) (7a-z). These Schiff base ligands (6a-f) and potential metal complexes were subjected to antifungal evaluation and algicidal effect.
\end{abstract}

\section{Materials and Methods}

\section{EXPERIMENTAL}

The chemicals and solvents were procured from Sigma-Aldrich, USA and Merck KGaA, Darmstadt, Germany and employed without any purification. 
RASĀYAN J. Chem.

Vol. 14 | No. 3 |2068-2076| July - September | 2021

\section{Characterization}

Gouy's method was used to determine the magnetic susceptibility at room temperature. FTIR spectra were recorded in the form of $\mathrm{KBr}$ disc between $4000-400 \mathrm{~cm}^{-1}$ on Perkin-Elmer FTIR spectrophotometer. The electronic absorption spectra of complexes and ligands were recorded between 200-900 nm in ethanol or DMF. ${ }^{1} \mathrm{H}$ NMR spectra were obtained on Bruker $400 \mathrm{MHz}$ NMR Spectrometer using TMS as an internal standard. Sulphur and halogen were estimated gravimetrically in the form of $\mathrm{BaSO}_{4}$ and $\mathrm{AgCl}$, respectively. The metal ions were estimated by standard methods. ${ }^{19}$

\section{Synthesis of Ligands}

4-Methylpiperidine-1-carbothiohydrazide (4) was synthesized using the reported method ${ }^{20,21}$ with few alterations (Scheme-I). The $N^{\prime}$-Substituted-4-methylpiperidine-1-carbothiohydrazides (6a-f) were synthesized by refluxing an equimolar amount of 4-Methylpiperidine-1-carbothiohydrazide (4) and corresponding aldehyde/ketone (5a-f) in ethanol for 2 hours. On cooling, the resultant obtained was filtered, washed with cold ethanol and recrystallized from ethanol (Scheme-II). The physicochemical and analytical data of synthesized ligands (6a-f) are summarized in Table-1.

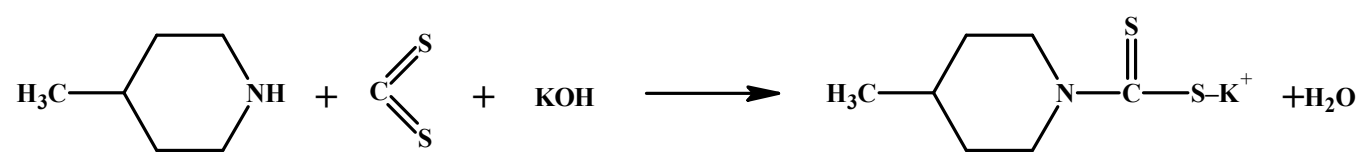

4-Methylpiperidine (1)

Potassium-4-methylpiperidine-dithiocarbamate (2)

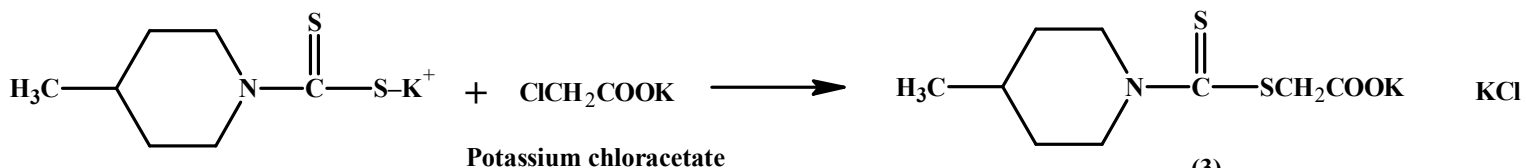

(2)

Potassium chloracetate

(3)

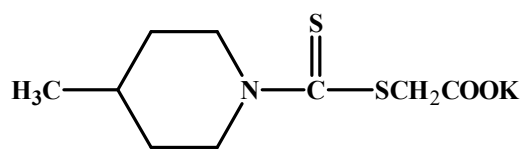

(3)

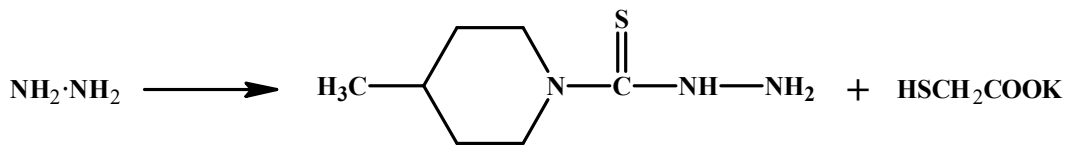

4-Methylpiperidine-1-carbothiohydrazide (4)

Scheme-I: Synthesis of 4-Methylpiperidine-1-carbothiohydrazide (4)

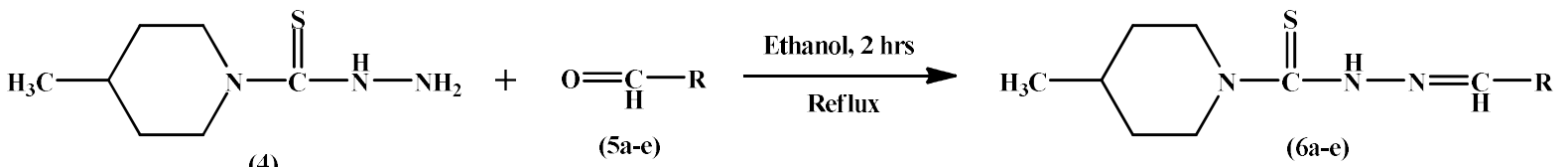

(4)

5a:<smiles>O=Cc1ccco1</smiles>

5b:<smiles>O=Cc1cccs1</smiles>

5c:<smiles>O=Cc1ccccc1</smiles>

5d:<smiles>O=Cc1ccccc1O</smiles>

5e:<smiles>O=C(CO)c1ccccc1</smiles>

Scheme-II: Synthesis of $N^{\prime}$-Substituted-4-methylpiperidine-1-carbothiohydrazides (6a-f) 
RASĀYAN J. Chem.

Vol. 14 | No. 3 |2068-2076| July - September | 2021

Table-1: Physico-analytical Data of Synthesized Ligands (6a-f)

\begin{tabular}{|c|c|c|c|c|c|c|}
\hline \multirow{2}{*}{ Ligands } & \multirow{2}{*}{ Color } & \multirow{2}{*}{$\begin{array}{l}\text { Melting } \\
\text { Point }\left({ }^{\circ} \mathrm{C}\right)\end{array}$} & \multicolumn{4}{|c|}{ Elemental Analysis: \% Found (Calcd.) } \\
\hline & & & $\mathrm{C}$ & $\mathrm{H}$ & $\mathrm{N}$ & $\mathrm{S}$ \\
\hline $\begin{array}{l}\text { 4-Methylpiperidine-1- } \\
\text { carbothiohydrazide (Hmpth, 4) }\end{array}$ & Flaky white & 100 & $\begin{array}{c}48.46 \\
(48.55)\end{array}$ & $\begin{array}{c}8.56 \\
(8.67)\end{array}$ & $\begin{array}{l}24.13 \\
(24.27)\end{array}$ & $\begin{array}{c}18.38 \\
(18.49)\end{array}$ \\
\hline $\begin{array}{c}N^{\prime} \text {-cyclohexylidene-4- } \\
\text { methylpiperidine-1- } \\
\text { carbothiohydrazide (Hcycmpth, 6f) }\end{array}$ & Cream & 149 & $\begin{array}{c}61.47 \\
(61.66)\end{array}$ & $\begin{array}{c}10.48 \\
(10.67)\end{array}$ & $\begin{array}{c}16.44 \\
(16.60)\end{array}$ & $\begin{array}{c}12.32 \\
(12.21)\end{array}$ \\
\hline $\begin{array}{l}N^{\prime} \text {-[(furan-2-yl)methylidene]-4- } \\
\text { methylpiperidine-1- } \\
\text { carbothiohydrazide (Hfmpth, 6a) }\end{array}$ & Cream & 164 & $\begin{array}{c}57.15 \\
(57.37)\end{array}$ & $\begin{array}{c}6.53 \\
(6.77)\end{array}$ & $\begin{array}{l}16.46 \\
(16.73)\end{array}$ & $\begin{array}{c}12.51 \\
(12.74)\end{array}$ \\
\hline $\begin{array}{c}\text { 4-methyl- } N^{\prime}-[(\text { thiophen-2- } \\
\text { yl)methylidene]piperidine-1- } \\
\text { carbothiohydrazide (Htalmpth, 6b) }\end{array}$ & Cream & 169 & $\begin{array}{c}53.68 \\
(53.93)\end{array}$ & $\begin{array}{c}6.18 \\
(6.36)\end{array}$ & $\begin{array}{c}15.50 \\
(15.73)\end{array}$ & $\begin{array}{l}23.73 \\
(23.97)\end{array}$ \\
\hline $\begin{array}{l}N^{\prime} \text {-benzylidene-4-methylpiperidine-1- } \\
\text { carbothiohydrazide (Hbenmpth, 6c) }\end{array}$ & Cream & 143 & $\begin{array}{c}64.11 \\
(64.36)\end{array}$ & $\begin{array}{c}7.08 \\
(7.27)\end{array}$ & $\begin{array}{c}15.83 \\
(15.49)\end{array}$ & $\begin{array}{c}11.92 \\
(11.86)\end{array}$ \\
\hline $\begin{array}{l}N^{\prime} \text {-[(2-hydroxyphenyl)methylidene]- } \\
\text { 4-methylpiperidine-1- } \\
\text { carbothiohydrazide (H2Salmpth, 6d) }\end{array}$ & White & 158 & $\begin{array}{c}60.48 \\
(60.64)\end{array}$ & $\begin{array}{c}6.62 \\
(6.85)\end{array}$ & $\begin{array}{l}14.95 \\
(14.76)\end{array}$ & $\begin{array}{c}11.31 \\
(11.15)\end{array}$ \\
\hline $\begin{array}{l}N^{\prime} \text {-(2-hydroxy-1-phenylethylidene)-4- } \\
\text { methylpiperidine-1- } \\
\text { carbothiohydrazide (H2apmpth, 6e) }\end{array}$ & White & 166 & $\begin{array}{c}61.62 \\
(61.85)\end{array}$ & $\begin{array}{c}6.92 \\
(6.81)\end{array}$ & $\begin{array}{c}14.22 \\
(14.43)\end{array}$ & $\begin{array}{c}10.73 \\
(10.99)\end{array}$ \\
\hline
\end{tabular}

\section{Synthesis of Metal Complexes}

Chromium Complexes [CrL $\left(\mathrm{H}_{2} \mathrm{O}\right)_{2} \mathrm{Cl}_{2}$ ] ( $\mathrm{LH}=$ Hcycmpth, Hfmpth, Htalmpth, Hbenmpth, $)$ and $\left[\mathrm{CrL}\left(\mathrm{H}_{2} \mathrm{O}\right)_{2} \mathrm{Cl}\right]\left(\mathrm{LH}_{2}=\mathrm{H}_{2} \mathrm{salmpth}, \mathrm{H}_{2}\right.$ apmpth $)$

An ethanolic solution of metal chloride $\left(\mathrm{CrCl}_{3} \cdot 6 \mathrm{H}_{2} \mathrm{O}, 40-50 \mathrm{~mL}\right)$ was added an ethanolic solution of respective ligands (molar ratio - 1:1) and the reaction mixture was refluxed for 3-4 hours. The $\mathrm{pH}$ was maintained at $\approx 7-8$ by adding sodium acetate solution. On cooling, the resultant metal complexes were filtered, washed with ethanol and dried over $\mathrm{CaCl}_{2}$ (anhydrous) at room temperature.

\section{Nickel and Zinc Metal Complexes}

(a) $\left[\mathrm{ML}_{2}\right]\left(\mathrm{M}=\mathrm{Ni}^{2+}\right.$ or $\mathrm{Zn}^{2+}$; $\mathrm{LH}=$ Hcycmpth, Hfmpth, Htalmpth, Hbenmpth) and $\left[\mathrm{M}(\mathrm{LH})_{2}\right](\mathrm{M}=$ $\mathrm{Ni}^{2+}$ or $\mathrm{Zn}^{2+}, \mathbf{L H}_{2}=\mathrm{H}_{2}$ salmpth, $\mathrm{H}_{2}$ apmpth)

To an ethanolic/aqueous solution of metal chloride $\left(\mathrm{NiCl}_{2} \cdot 6 \mathrm{H}_{2} \mathrm{O} / \mathrm{ZnCl}_{2} \cdot \mathrm{H}_{2} \mathrm{O}, 40-50 \mathrm{~mL}\right)$ was added hot ethanolic solution of appropriate ligand (1:2) slowly with continuous stirring and refluxed for 1-2 hours. On cooling, metal complexes were separated gradually. In the case of $\mathrm{Zn}$ (II) complexes, the complexes separated on adding an equal volume of water. The products were collected on a filter paper, washed with aqueous ethanol and dried over anhydrous $\mathrm{CaCl}_{2}$ and finally vacuum dried.

\section{[ML( $\left.\left.\mathbf{N H}_{3}\right)\right]\left(\mathbf{M}=\mathbf{N i}^{2+}\right.$ or $\mathbf{Z n}^{2+} ; \mathbf{L H}_{2}=\mathbf{H}_{2}$ salmpth, $\mathbf{H}_{2}$ apmpth $)$}

An aqueous solution of appropriate metal acetate $\left[\mathrm{Ni}(\mathrm{OAc})_{2} \cdot 4 \mathrm{H}_{2} \mathrm{O} ; \mathrm{Zn}(\mathrm{OAc})_{2} \cdot 2 \mathrm{H}_{2} \mathrm{O}, 40-50 \mathrm{~mL}\right]$ was allowed to reflux for 2 hours with 40-50 mL ethanolic solution of appropriate ligand (1:1) in the presence of $15 \%$ of $\mathrm{NH}_{4} \mathrm{OH}$ and $\mathrm{pH}$ was maintained at $\approx 9$. The resulting metal complexes were collected on a filter paper, washed with aqueous ethanol and dried over anhydrous $\mathrm{CaCl}_{2}$ and finally vacuum dried.

\section{[ML(Py)] (M=Ni ${ }^{2+}$ or $\mathrm{Zn}^{2+} ; \mathbf{L H}_{2}=\mathrm{H}_{2} \mathrm{Salmpth}, \mathrm{H}_{2}$ apmpth)}

An aqueous solution of metal chloride $(0.0015 \mathrm{~mol}, 50 \mathrm{~mL})$ was treated with $3-4 \mathrm{~mL}$ of pyridine and the resultant solution was added in hot ethanolic solution $(50 \mathrm{~mL})$ of appropriate ligand $(0.0015 \mathrm{~mol})$ and refluxed for 1-2 hours. On cooling, the metal complexes were separated. The solid product was washed with aqueous ethanol and dried with $\mathrm{KOH}+\mathrm{CaCl}_{2}$. Physico-analytical data of the synthesized complexes are given in Table-2. 
RASĀYAN J. Chem.

Vol. 14 | No. 3 |2068-2076| July - September | 2021

Table-2: Physico-analytical Data of Schiff Base Metal Complexes derived from $N^{\prime}$-substituted-4-methylpiperidine1-carbothiohydrazides (6a-f)

\begin{tabular}{|c|c|c|c|c|c|c|c|c|}
\hline \multirow{2}{*}{ Complexes } & \multirow{2}{*}{ Color } & \multicolumn{5}{|c|}{ Elemental Analysis: \% Found (Calc.) } & \multirow{2}{*}{$\begin{array}{c}\mu \mathrm{eff} \\
\text { (B.M.) }\end{array}$} & \multirow{2}{*}{$\begin{array}{l}\Lambda_{\mathrm{M}}\left(\mathrm{ohm}^{-1}\right. \\
\left.\mathrm{cm}^{2} \mathrm{~mol}^{-1}\right)\end{array}$} \\
\hline & & $\mathrm{M}$ & $\mathrm{C}$ & $\mathrm{H}$ & $\mathrm{N}$ & $\mathrm{S}$ & & \\
\hline$\left[\mathrm{Cr}(\mathrm{cycmpth})\left(\mathrm{H}_{2} \mathrm{O}\right)_{2} \mathrm{Cl}_{2}\right], 7 \mathrm{a}$ & Green & $\begin{array}{c}12.47 \\
(12.68) \\
\end{array}$ & $\begin{array}{c}37.94 \\
(37.81)\end{array}$ & $\begin{array}{c}5.92 \\
(5.89)\end{array}$ & $\begin{array}{c}10.11 \\
(10.24)\end{array}$ & $\begin{array}{c}7.95 \\
(7.80) \\
\end{array}$ & 3.87 & 5.71 \\
\hline$\left[\mathrm{Cr}(\mathrm{fmpth})\left(\mathrm{H}_{2} \mathrm{O}\right)_{2} \mathrm{Cl}_{2}\right], 7 \mathrm{~b}$ & Dark green & $\begin{array}{c}12.50 \\
(12.71) \\
\end{array}$ & $\begin{array}{c}34.88 \\
(34.73) \\
\end{array}$ & $\begin{array}{c}4.69 \\
(4.88) \\
\end{array}$ & $\begin{array}{c}10.11 \\
(10.26)\end{array}$ & $\begin{array}{c}7.66 \\
(7.82) \\
\end{array}$ & 3.90 & 8.33 \\
\hline$\left[\mathrm{Cr}\right.$ (talmpth) $\left.\left(\mathrm{H}_{2} \mathrm{O}\right)_{2} \mathrm{Cl}_{2}\right], 7 \mathrm{c}$ & Dark Green & $\begin{array}{c}12.08 \\
(12.23)\end{array}$ & $\begin{array}{c}33.67 \\
(33.88)\end{array}$ & $\begin{array}{c}4.52 \\
(4.70)\end{array}$ & $\begin{array}{c}9.68 \\
(9.88)\end{array}$ & $\begin{array}{c}14.88 \\
(14.95) \\
\end{array}$ & 3.89 & 7.15 \\
\hline$\left[\mathrm{Cr}(\right.$ benmpth $\left.)\left(\mathrm{H}_{2} \mathrm{O}\right)_{2} \mathrm{Cl}_{2}\right], 7 \mathrm{~d}$ & Dark Green & $\begin{array}{c}12.18 \\
(12.38)\end{array}$ & $\begin{array}{c}39.90 \\
(39.80)\end{array}$ & $\begin{array}{c}5.28 \\
(5.17)\end{array}$ & $\begin{array}{c}9.73 \\
(9.62)\end{array}$ & $\begin{array}{c}7.42 \\
(7.61)\end{array}$ & 3.88 & 6.29 \\
\hline$\left[\mathrm{Cr}(\mathrm{salmpth})\left(\mathrm{H}_{2} \mathrm{O}\right)_{2} \mathrm{Cl}\right], 7 \mathrm{e}$ & Brown & $\begin{array}{c}12.73 \\
(12.98) \\
\end{array}$ & $\begin{array}{c}41.76 \\
(41.94) \\
\end{array}$ & $\begin{array}{c}5.53 \\
(5.34) \\
\end{array}$ & $\begin{array}{c}10.31 \\
(10.44) \\
\end{array}$ & $\begin{array}{c}7.76 \\
(7.69) \\
\end{array}$ & 3.89 & 7.74 \\
\hline$\left[\mathrm{Cr}(\mathrm{apmpth})\left(\mathrm{H}_{2} \mathrm{O}\right)_{2} \mathrm{Cl}\right], 7 \mathrm{f}$ & Brown & $\begin{array}{c}12.32 \\
(12.54) \\
\end{array}$ & $\begin{array}{c}43.22 \\
(43.12) \\
\end{array}$ & $\begin{array}{c}5.83 \\
(5.73) \\
\end{array}$ & $\begin{array}{c}9.97 \\
(9.83) \\
\end{array}$ & $\begin{array}{c}7.53 \\
(7.72) \\
\end{array}$ & 3.90 & 7.37 \\
\hline$\left[\mathrm{Ni}(\text { cycmpth })_{2}\right], 7 \mathrm{~g}$ & Brown & $\begin{array}{c}10.46 \\
(10.51) \\
\end{array}$ & $\begin{array}{c}55.40 \\
(55.61) \\
\end{array}$ & $\begin{array}{c}7.23 \\
(7.48) \\
\end{array}$ & $\begin{array}{c}14.75 \\
(14.97) \\
\end{array}$ & $\begin{array}{c}11.18 \\
(11.40) \\
\end{array}$ & Diamag. & 6.53 \\
\hline [Zn(cycmpth $\left.)_{2}\right], 7 \mathrm{~h}$ & Light Yellow & $\begin{array}{c}11.25 \\
(11.46) \\
\end{array}$ & $\begin{array}{c}54.88 \\
(54.72) \\
\end{array}$ & $\begin{array}{c}7.26 \\
(7.40) \\
\end{array}$ & $\begin{array}{c}14.65 \\
(14.81) \\
\end{array}$ & $\begin{array}{c}11.11 \\
(11.28) \\
\end{array}$ & Diamag. & 5.74 \\
\hline$\left[\mathrm{Ni}(\mathrm{fmpth})_{2}\right], 7 \mathrm{i}$ & Brown & $\begin{array}{c}10.38 \\
(10.55)\end{array}$ & $\begin{array}{c}51.33 \\
(51.52)\end{array}$ & $\begin{array}{c}5.57 \\
(5.72)\end{array}$ & $\begin{array}{l}14.88 \\
15.02 \\
\end{array}$ & $\begin{array}{c}11.26 \\
(11.44)\end{array}$ & Diamag. & 7.45 \\
\hline$\left[\mathrm{Zn}(\mathrm{fmpth})_{2}\right], 7 \mathrm{j}$ & Yellow & $\begin{array}{c}11.31 \\
(11.50)\end{array}$ & $\begin{array}{c}50.73 \\
(50.97)\end{array}$ & $\begin{array}{c}5.48 \\
(5.66)\end{array}$ & $\begin{array}{c}14.94 \\
(14.86)\end{array}$ & $\begin{array}{c}11.20 \\
(11.32)\end{array}$ & Diamag. & 8.92 \\
\hline [Ni(talmpth) $\left.)_{2}\right], 7 \mathrm{k}$ & Brown & $\begin{array}{c}9.77 \\
(9.98) \\
\end{array}$ & $\begin{array}{c}48.55 \\
(48.73) \\
\end{array}$ & $\begin{array}{c}5.24 \\
(5.41) \\
\end{array}$ & $\begin{array}{c}14.05 \\
(14.21) \\
\end{array}$ & $\begin{array}{c}21.44 \\
(21.65) \\
\end{array}$ & Diamag. & 6.28 \\
\hline [Zn(talmpth) $\left.)_{2}\right], 71$ & Yellow & $\begin{array}{c}10.63 \\
(10.88) \\
\end{array}$ & $\begin{array}{c}48.11 \\
(48.24) \\
\end{array}$ & $\begin{array}{c}5.17 \\
(5.36) \\
\end{array}$ & $\begin{array}{c}14.15 \\
(14.07) \\
\end{array}$ & $\begin{array}{c}21.30 \\
(21.44) \\
\end{array}$ & Diamag. & 7.54 \\
\hline [Ni(benmpth) 2$], 7 \mathrm{~m}$ & Orange Red & $\begin{array}{c}10.08 \\
(10.22)\end{array}$ & $\begin{array}{c}58.06 \\
(58.23) \\
\end{array}$ & $\begin{array}{c}5.71 \\
(5.89)\end{array}$ & $\begin{array}{c}14.32 \\
(14.55)\end{array}$ & $\begin{array}{c}10.86 \\
(10.79) \\
\end{array}$ & Diamag. & 4.90 \\
\hline$\left[\mathrm{Zn}(\text { benmpth })_{2}\right], 7 \mathrm{n}$ & $\begin{array}{l}\text { Orange } \\
\text { Yellow }\end{array}$ & $\begin{array}{c}10.93 \\
(11.04)\end{array}$ & $\begin{array}{c}57.51 \\
(57.63)\end{array}$ & $\begin{array}{c}5.61 \\
(5.83)\end{array}$ & $\begin{array}{c}14.26 \\
(14.10)\end{array}$ & $\begin{array}{c}10.76 \\
(10.67)\end{array}$ & Diamag. & 10.52 \\
\hline [Ni(HSalmpth) $\left.)_{2}\right], 7 \mathrm{o}$ & $\begin{array}{l}\text { Reddish } \\
\text { Brown }\end{array}$ & $\begin{array}{c}9.44 \\
(9.68) \\
\end{array}$ & $\begin{array}{l}54.97 \\
55.07 \\
\end{array}$ & $\begin{array}{c}5.33 \\
(5.58) \\
\end{array}$ & $\begin{array}{c}13.58 \\
(13.79) \\
\end{array}$ & $\begin{array}{c}10.32 \\
(10.50) \\
\end{array}$ & Diamag. & 6.4 \\
\hline [Zn(HSalmpth) $\left.)_{2}\right], 7 \mathrm{p}$ & Yellow & $\begin{array}{c}10.32 \\
(10.56) \\
\end{array}$ & $\begin{array}{c}55.25 \\
(55.63) \\
\end{array}$ & $\begin{array}{c}5.26 \\
(5.52)\end{array}$ & $\begin{array}{c}13.48 \\
(13.65) \\
\end{array}$ & $\begin{array}{c}10.27 \\
(10.40) \\
\end{array}$ & Diamag. & 7.36 \\
\hline$\left[\mathrm{Ni}(\text { Hapmpth) })_{2}\right], 7 \mathrm{q}$ & Brick Red & $\begin{array}{c}9.12 \\
(9.26)\end{array}$ & $\begin{array}{c}56.28 \\
(56.51) \\
\end{array}$ & $\begin{array}{c}5.64 \\
(5.96)\end{array}$ & $\begin{array}{c}12.93 \\
(13.18)\end{array}$ & $\begin{array}{c}9.88 \\
(10.04)\end{array}$ & Diamag. & 6.50 \\
\hline [Zn(Hapmpth) 2$], 7 \mathrm{r}$ & Yellow & $\begin{array}{c}9.87 \\
(10.01)\end{array}$ & $\begin{array}{c}55.74 \\
(55.98)\end{array}$ & $\begin{array}{c}6.31 \\
(6.53)\end{array}$ & $\begin{array}{c}12.87 \\
(12.76)\end{array}$ & $\begin{array}{c}9.73 \\
(9.65)\end{array}$ & Diamag. & 7.75 \\
\hline$\left[\mathrm{Ni}(\right.$ Salmpth $\left.)\left(\mathrm{NH}_{3}\right)\right], 7 \mathrm{~s}$ & Red & $\begin{array}{c}16.63 \\
(16.80) \\
\end{array}$ & $\begin{array}{c}47.67 \\
(47.86) \\
\end{array}$ & $\begin{array}{c}5.48 \\
(5.69) \\
\end{array}$ & $\begin{array}{c}15.74 \\
(15.65) \\
\end{array}$ & $\begin{array}{c}8.96 \\
(8.91) \\
\end{array}$ & Diamag. & 9.63 \\
\hline$\left[\mathrm{Zn}(\right.$ Salmpth $\left.)\left(\mathrm{NH}_{3}\right)\right], 7 \mathrm{t}$ & Yellow & $\begin{array}{c}18.03 \\
(18.20) \\
\end{array}$ & $\begin{array}{c}47.86 \\
(47.05) \\
\end{array}$ & $\begin{array}{c}5.47 \\
(5.60) \\
\end{array}$ & $\begin{array}{c}15.47 \\
(15.68) \\
\end{array}$ & $\begin{array}{c}8.68 \\
(8.96) \\
\end{array}$ & Diamag. & 11.31 \\
\hline [Ni(apmpth) $\left.\left(\mathrm{NH}_{3}\right)\right], 7 \mathrm{u}$ & $\begin{array}{l}\text { Reddish } \\
\text { Brown }\end{array}$ & $\begin{array}{c}15.98 \\
(16.06)\end{array}$ & $\begin{array}{c}49.14 \\
(49.31)\end{array}$ & $\begin{array}{c}5.93 \\
(6.02)\end{array}$ & $\begin{array}{c}15.17 \\
(15.34)\end{array}$ & $\begin{array}{c}8.58 \\
(8.46)\end{array}$ & Diamag. & 10.85 \\
\hline$\left[\mathrm{Zn}(\mathrm{apmpth})\left(\mathrm{NH}_{3}\right)\right], 7 \mathrm{v}$ & Yellow & $\begin{array}{c}17.37 \\
(17.52) \\
\end{array}$ & $\begin{array}{c}48.36 \\
(48.51) \\
\end{array}$ & $\begin{array}{c}5.68 \\
(5.92)\end{array}$ & $\begin{array}{c}14.92 \\
(14.99)\end{array}$ & $\begin{array}{c}8.47 \\
(8.62) \\
\end{array}$ & Diamag. & 11.26 \\
\hline [Ni(Salmpth)Py], 7w & Red & $\begin{array}{c}14.11 \\
(14.28)\end{array}$ & $\begin{array}{c}54.98 \\
(55.02)\end{array}$ & $\begin{array}{c}5.11 \\
(5.32)\end{array}$ & $\begin{array}{c}13.34 \\
(13.55)\end{array}$ & $\begin{array}{c}7.51 \\
(7.74)\end{array}$ & Diamag. & 8.28 \\
\hline [Ni(apmpth)Py], 7x & $\begin{array}{l}\text { Reddish } \\
\text { Brown }\end{array}$ & $\begin{array}{c}13.62 \\
(13.81)\end{array}$ & $\begin{array}{c}56.03 \\
(56.20)\end{array}$ & $\begin{array}{c}5.41 \\
(5.62)\end{array}$ & $\begin{array}{c}12.92 \\
(13.01)\end{array}$ & $\begin{array}{c}7.23 \\
(7.49)\end{array}$ & Diamag. & 9.57 \\
\hline [Zn(Salmpth)Py], 7y & Light Yellow & $\begin{array}{c}15.33 \\
(15.51)\end{array}$ & $\begin{array}{c}54.24 \\
(54.41) \\
\end{array}$ & $\begin{array}{c}5.09 \\
(5.25)\end{array}$ & $\begin{array}{c}13.12 \\
(13.36)\end{array}$ & $\begin{array}{c}7.45 \\
(7.63) \\
\end{array}$ & Diamag. & 10.12 \\
\hline [Zn(apmpth)Py], 7z & Yellow & $\begin{array}{c}14.88 \\
(14.71)\end{array}$ & $\begin{array}{c}55.20 \\
(55.42)\end{array}$ & $\begin{array}{c}5.36 \\
(5.54)\end{array}$ & $\begin{array}{c}12.64 \\
(12.93)\end{array}$ & $\begin{array}{c}7.18 \\
(7.11)\end{array}$ & Diamag. & 6.32 \\
\hline
\end{tabular}


RASĀYAN J. Chem.

Vol. 14 | No. 3 |2068-2076| July - September | 2021

Table-3: Electronic Spectral Data of Schiff Base Metal Complexes derived from $N^{\prime}$-substituted-4-methylpiperidine1-carbothiohydrazides (6a-f)

\begin{tabular}{|c|c|c|}
\hline Complexes & Electronic Bond Positions & Assingments \\
\hline$\left[\mathrm{Cr}(\mathrm{cycmpth})_{2}\left(\mathrm{H}_{2} \mathrm{O}\right)_{2} \mathrm{Cl}_{2}\right]$ & $310 \mathrm{w}, 425 \mathrm{~s}, 568 \mathrm{sbr}$ & ${ }^{4} \mathrm{~A}_{2} \mathrm{~g}(\mathrm{~F}) \rightarrow{ }^{4} \mathrm{~T}_{1 \mathrm{~g}}(\mathrm{P}) ;{ }^{4} \mathrm{~A}_{2 \mathrm{~g}}(\mathrm{~F})-{ }^{4} \mathrm{~T}_{1 \mathrm{~g}}(\mathrm{~F}) ;{ }^{4} \mathrm{~A}_{2 \mathrm{~g}}(\mathrm{~F}) \rightarrow{ }^{4} \mathrm{~T}_{2 \mathrm{~g}}(\mathrm{P})$ \\
\hline$\left[\mathrm{Cr}(\mathrm{fmpth})_{2}\left(\mathrm{H}_{2} \mathrm{O}\right)_{2} \mathrm{Cl}_{2}\right]$ & $325 \mathrm{w}, 440 \mathrm{sh}, 580 \mathrm{sbr}$ & ${ }^{4} \mathrm{~A}_{2} \mathrm{~g}(\mathrm{~F}) \rightarrow{ }^{4} \mathrm{~T}_{1 \mathrm{~g}}(\mathrm{P}) ;{ }^{4} \mathrm{~A}_{2 \mathrm{~g}}(\mathrm{~F})-{ }^{4} \mathrm{~T}_{1 \mathrm{~g}}(\mathrm{~F}) ;{ }^{4} \mathrm{~A}_{2 \mathrm{~g}}(\mathrm{~F}) \rightarrow{ }^{4} \mathrm{~T}_{2 \mathrm{~g}}(\mathrm{P})$ \\
\hline$\left[\mathrm{Cr}(\text { talmpth })_{2}\left(\mathrm{H}_{2} \mathrm{O}\right)_{2} \mathrm{Cl}_{2}\right]$ & $315 \mathrm{w}, 432 \mathrm{~s}, 570 \mathrm{sbr}$ & ${ }^{4} \mathrm{~A}_{2} \mathrm{~g}(\mathrm{~F}) \rightarrow{ }^{4} \mathrm{~T}_{1 \mathrm{~g}}(\mathrm{P}) ;{ }^{4} \mathrm{~A}_{2 \mathrm{~g}}(\mathrm{~F})-{ }^{4} \mathrm{~T}_{1 \mathrm{~g}}(\mathrm{~F}) ;{ }^{4} \mathrm{~A}_{2 \mathrm{~g}}(\mathrm{~F}) \rightarrow{ }^{4} \mathrm{~T}_{2 \mathrm{~g}}(\mathrm{P})$ \\
\hline$\left[\mathrm{Cr}(\text { benmpth })_{2}\left(\mathrm{H}_{2} \mathrm{O}\right)_{2} \mathrm{Cl}_{2}\right]$ & $318 \mathrm{w}, 445 \mathrm{~s}, 575 \mathrm{sbr}$ & ${ }^{4} \mathrm{~A}_{2} \mathrm{~g}(\mathrm{~F}) \rightarrow{ }^{4} \mathrm{~T}_{1 \mathrm{~g}}(\mathrm{P}) ;{ }^{4} \mathrm{~A}_{2 \mathrm{~g}}(\mathrm{~F})-{ }^{4} \mathrm{~T}_{1 \mathrm{~g}}(\mathrm{~F}) ;{ }^{4} \mathrm{~A}_{2 \mathrm{~g}}(\mathrm{~F}) \rightarrow{ }^{4} \mathrm{~T}_{2 \mathrm{~g}}(\mathrm{P})$ \\
\hline$\left[\mathrm{Cr}(\right.$ salmpth $\left.)\left(\mathrm{H}_{2} \mathrm{O}\right)_{2} \mathrm{Cl}\right]$ & $320 \mathrm{w}, 452 \mathrm{~s}, 582 \mathrm{sbr}$ & ${ }^{4} \mathrm{~A}_{2} \mathrm{~g}(\mathrm{~F}) \rightarrow{ }^{4} \mathrm{~T}_{1 \mathrm{~g}}(\mathrm{P}) ;{ }^{4} \mathrm{~A}_{2 \mathrm{~g}}(\mathrm{~F})-{ }^{4} \mathrm{~T}_{1 \mathrm{~g}}(\mathrm{~F}) ;{ }^{4} \mathrm{~A}_{2 \mathrm{~g}}(\mathrm{~F}) \rightarrow{ }^{4} \mathrm{~T}_{2 \mathrm{~g}}(\mathrm{P})$ \\
\hline$\left[\mathrm{Cr}(\right.$ apmpth $\left.)\left(\mathrm{H}_{2} \mathrm{O}\right)_{2} \mathrm{Cl}\right]$ & $325 \mathrm{w}, 455 \mathrm{~s}, 585 \mathrm{sbr}$ & ${ }^{4} \mathrm{~A}_{2} \mathrm{~g}(\mathrm{~F}) \rightarrow{ }^{4} \mathrm{~T}_{1 \mathrm{~g}}(\mathrm{P}) ;{ }^{4} \mathrm{~A}_{2 \mathrm{~g}}(\mathrm{~F})-{ }^{4} \mathrm{~T}_{1 \mathrm{~g}}(\mathrm{~F}) ;{ }^{4} \mathrm{~A}_{2 \mathrm{~g}}(\mathrm{~F}) \rightarrow{ }^{4} \mathrm{~T}_{2 \mathrm{~g}}(\mathrm{P})$ \\
\hline$\left[\mathrm{Ni}(\text { cycmpth })_{2}\right]$ & $290 \mathrm{~ms}, 440 \mathrm{sh}$ & $\pi-\pi^{*} ;{ }^{1} \mathrm{~A}_{1 \mathrm{~g}} \rightarrow{ }^{1} \mathrm{~B}_{1 \mathrm{~g}}$ \\
\hline$\left[\mathrm{Ni}(\mathrm{fmpth})_{2}\right]$ & $375 \mathrm{sbr}, 440 \mathrm{br}, 552 \mathrm{~m}$ & $\mathrm{C}-\mathrm{T} ; \mathrm{A}_{1 \mathrm{~g}} \rightarrow{ }^{1} \mathrm{~B}_{1 \mathrm{~g}} ;{ }^{1} \mathrm{~A}_{1 \mathrm{~g}} \rightarrow{ }^{1} \mathrm{~A}_{2 \mathrm{~g}}$ \\
\hline$\left[\mathrm{Ni}(\text { talmpth })_{2}\right]$ & $370 \mathrm{sbr}, 435 \mathrm{br}, 545 \mathrm{~m}$ & $\mathrm{C}-\mathrm{T} ;{ }^{1} \mathrm{~A}_{1 \mathrm{~g}} \rightarrow{ }^{1} \mathrm{~B}_{1 \mathrm{~g}} ;{ }^{1} \mathrm{~A}_{1 \mathrm{~g}} \rightarrow{ }^{1} \mathrm{~A}_{2 \mathrm{~g}}$ \\
\hline [Ni(benmpth) $)_{2}$ & $378 \mathrm{br}, 450 \mathrm{~m}, 560 \mathrm{~m}$ & $\mathrm{C}-\mathrm{T} ;{ }^{1} \mathrm{~A}_{1 \mathrm{~g}} \rightarrow{ }^{1} \mathrm{~B}_{1 \mathrm{~g}} ;{ }^{1} \mathrm{~A}_{1 \mathrm{~g}} \rightarrow{ }^{1} \mathrm{~A}_{2 \mathrm{~g}}$ \\
\hline$\left[\mathrm{Ni}(\text { HSalmpth })_{2}\right]$ & $368 \mathrm{~b}, 455 \mathrm{mb}, 565 \mathrm{~m}$ & $\mathrm{C}-\mathrm{T} ;{ }^{1} \mathrm{~A}_{1 \mathrm{~g}} \rightarrow{ }^{1} \mathrm{~B}_{1 \mathrm{~g}} ;{ }^{1} \mathrm{~A}_{1 \mathrm{~g}} \rightarrow^{1} \mathrm{~A}_{2 \mathrm{~g}}$ \\
\hline$\left[\mathrm{Ni}(\text { Hapmpth })_{2}\right]$ & $272 \mathrm{~s}, 370 \mathrm{br}, 460 \mathrm{sh}, 567 \mathrm{~m}$ & $\pi-\pi^{*} ; \mathrm{C}-\mathrm{T} ;{ }^{1 \mathrm{~A}} 1 \mathrm{~g} \rightarrow 1 \mathrm{~B}{ }_{1 \mathrm{~g}} ;{ }^{1} \mathrm{~A}_{1 \mathrm{~g}} \rightarrow{ }^{1} \mathrm{~A}_{2 \mathrm{~g}}$ \\
\hline$\left[\mathrm{Ni}(\mathrm{Salmpth})\left(\mathrm{NH}_{3}\right)\right]$ & $262 \mathrm{~s}, 370 \mathrm{sbr}, 450 \mathrm{sh}$ & $\pi-\pi^{*} ; \mathrm{C}-\mathrm{T} ;{ }^{1} \mathrm{~A}_{1 \mathrm{~g}} \rightarrow{ }^{1} \mathrm{~B}_{1 \mathrm{~g}}$ \\
\hline$\left[\mathrm{Ni}(\right.$ apmpth $\left.)\left(\mathrm{NH}_{3}\right)\right]$ & $260 \mathrm{~s}, 365 \mathrm{sbr}, 447 \mathrm{sh}$ & $\pi-\pi^{*} ; \mathrm{C}-\mathrm{T} ;{ }^{1} \mathrm{~A}_{1 \mathrm{~g}} \rightarrow{ }^{1} \mathrm{~B}_{1 \mathrm{~g}}$ \\
\hline [Ni(salmpth)(Py) & $265 \mathrm{~s}, 372 \mathrm{sbr}, 450 \mathrm{sh}$ & $\pi-\pi^{*} ; \mathrm{C}-\mathrm{T} ;{ }^{1} \mathrm{~A}_{1 \mathrm{~g}} \rightarrow{ }^{1} \mathrm{~B}_{1 \mathrm{~g}}$ \\
\hline [Ni(apmpth)(Py) & $262 \mathrm{~s}, 368 \mathrm{sbr}, 445 \mathrm{sh}$ & $\pi-\pi^{*} ; \mathrm{C}-\mathrm{T} ;{ }^{1} \mathrm{~A}_{1 \mathrm{~g}} \rightarrow{ }^{1} \mathrm{~B}_{1 \mathrm{~g}}$ \\
\hline
\end{tabular}

( $\mathrm{Sh}=$ shoulder, $\mathrm{m}=$ medium, $\mathrm{s}=$ strong, $\mathrm{sbr}=$ strong and broad)

\section{RESULTS AND DISCUSSION}

The analytical data recorded in Table-2 were found fully consistent with the composition of the complexes synthesized. Thermogravimetric analysis shows that all the synthesized complexes are stable below $180-190{ }^{\circ} \mathrm{C}$. The complexes are lesser soluble in ordinary organic solvents and are much soluble in DMSO, DMF and dioxane. Molar conductance of all the complexes was found in the range 5-15 $\Omega^{-1} \mathrm{~cm}^{2}$ mol $^{-1}$ displaying their non-ionic nature. ${ }^{22}$ The $\mathrm{Cr}(\mathrm{III})$ complexes have magnetic moments value between 3.87-3.91 B.M. indicating their octahedral geometry. The diamagnetism of all $\mathrm{Ni}(\mathrm{II})$ complexes indicates their square planar geometry ${ }^{23}$. The preference for tetrahedral stereochemistry for complexes of $d^{10}$ electronic system and observed metal-ligand stoichiometry indicate the tetrahedral structure for $\mathrm{Zn}$ (II) complexes, which were established as diamagnetic. ${ }^{24}$

\section{Electronic Spectral Studies}

The chemical spectra of all Cr(III) complexes were obtained in DMSO. The complexes exhibited bands at $16806-18518 \mathrm{~cm}^{-1}(595-540 \mathrm{~nm}), 22831-23539 \mathrm{~cm}^{-1}(438-425 \mathrm{~nm})$ and $31250-32573 \mathrm{~cm}^{-1}(320-307 \mathrm{~nm})$ which may be assigned to ${ }^{4} \mathrm{~A}_{2} \mathrm{~g}(\mathrm{~F}) \rightarrow{ }^{4} \mathrm{~T}_{2} \mathrm{~g}(\mathrm{~F})\left(v_{1}\right),{ }^{4} \mathrm{~A}_{2} \mathrm{~g}(\mathrm{~F}) \rightarrow{ }^{4} \mathrm{~T}_{1} \mathrm{~g}(\mathrm{~F})\left(\mathrm{v}_{2}\right)$ and ${ }^{4} \mathrm{~A}_{2} \mathrm{~g}(\mathrm{~F}) \rightarrow{ }^{4} \mathrm{~T}_{1} \mathrm{~g}(\mathrm{P})\left(v_{3}\right)$ transitions sequentially in order of increasing energy. Out of these three spin-allowed $d-d$ transitions, the one of lowest energy is a direct measure of the crystal field splitting $\Delta$ or $10 \mathrm{Dq}$. These values are in better compliance with the reported values for octahedral $\mathrm{Cr}$ (III) complexes ${ }^{25,26}$. The solid reflectance spectra of $\mathrm{Ni}$ (II) complexes below $400 \mathrm{~nm}$ display strong absorptions attributed to charge transfer band or ligand absorptions. A weak band or shoulder in the region $420-480 \mathrm{~nm}$ and 500-600 nm are attributed to ${ }^{1} \mathrm{~A}_{1} \mathrm{~g} \rightarrow$ ${ }^{1} \mathrm{~B}_{1} \mathrm{~g}$ and ${ }^{1} \mathrm{~A}_{1} \mathrm{~g} \rightarrow{ }^{1} \mathrm{~A}_{2} \mathrm{~g}$ transitions respectively in a planner arrangement of $\mathrm{Ni}(\mathrm{II})$ atom geometry ${ }^{23}$. The broadband of thioamide ligands was observed in the regions $\approx 365 \mathrm{~nm}$ and suffered a blue shift in the complexes due to thioenolization, which broadens considerably due to overlap of ligands to metal charge transfer transitions ${ }^{27}$ (Table-3).

\section{FTIR Spectral Studies}

The IR spectra of ligands exhibited bands at $1625 \pm 5 \mathrm{~cm}^{-1}$, which is due to $\mathrm{v}_{\mathrm{C}=\mathrm{N}}$ of the azomethine group. The same band is observed at a lower frequency by $15-10 \mathrm{~cm}^{-1}$ as a strong band in all the complexes of $\mathrm{Cr}(\mathrm{III}), \mathrm{Ni}(\mathrm{II})$ and $\mathrm{Zn}$ (II) attributed to the coordination through azomethine nitrogen. It is further confirmed by the appearance of azine chromophore $(>\mathrm{C}=\mathrm{N}-\mathrm{N}=\mathrm{C}<)$ around $1605-1600 \mathrm{~cm}^{-1}$ in the 
complexes. The characteristic phenolic $v_{(\mathrm{O}-\mathrm{H})}$ band due to the presence of -OH group at ortho position of ligands $\left(\mathrm{H}_{2} \mathrm{Salmpth}\right)$ and $\left(\mathrm{H}_{2}\right.$ ampth $)$ was observed at 3293-3180 $\mathrm{cm}^{-1}$, disappeared in the monoligated complexes of $\mathrm{Cr}(\mathrm{III}), \mathrm{Ni}(\mathrm{II})$ and $\mathrm{Zn}(\mathrm{II})$ having compositions [ $\mathrm{CrL}\left(\mathrm{H}_{2} \mathrm{O}\right)_{2} \mathrm{Cl}$ ], [ML(Py)], [ML( $\left.\mathrm{NH}_{3}\right)$ ] $\left(\mathrm{M}=\mathrm{Ni}^{2+}\right.$ or $\mathrm{Zn}^{2+}, \mathrm{LH}_{2}=\mathrm{H}_{2}$ Salmpth, $\mathrm{H}_{2}$ apmth) suggesting the coordination by the phenolic oxygen after deprotonation. This broadband is intact in bischelates of $\mathrm{Ni}(\mathrm{II})$ and $\mathrm{Zn}$ (II) having compositions [ $\left.\mathrm{ML}_{2}\right]$ and $\left[\mathrm{M}\left(\mathrm{LH}_{2}\right)\left(\mathrm{LH}=\mathrm{Hcycmpth}\right.\right.$, Hfmpth,Htalmpth, Hbenmpth and $\mathrm{LH}_{2}=\mathrm{H}_{2}$ Salmpth, $\mathrm{H}_{2}$ ampth $)$ and attributed to uncoordinated $-\mathrm{OH}$ and involved in $\mathrm{H}$-bonding. In all the aqua complexes of $\mathrm{Cr}(\mathrm{III})$ having composition [ML $\left.\left(\mathrm{H}_{2} \mathrm{O}\right)\right]\left(\mathrm{LH}_{2}=\mathrm{H}_{2}\right.$ Salmpth, $\mathrm{H}_{2}$ apampth $)$ a broad bond appeared in the range of $3400-3300 \mathrm{~cm}^{-1}$ showing the existence of water. The rocking band of $\mathrm{H}_{2} \mathrm{O}$ is not observed for coordinated water which indicates the lattice nature of $\mathrm{H}_{2} \mathrm{O}$ in the complexes. ${ }^{28}$ Drastic conditions required to dehydrate these complexes also confirm a structure containing water molecules in these complexes. The absence of any band in the region of $2600-2500 \mathrm{~cm}^{-1}$ due to $v_{(\mathrm{SH})}$ shows that all the ligands exist in thio-keto form, which is also supported by ${ }^{1} \mathrm{H}$ NMR studies. The IR absorption bands of $v_{\mathrm{NH}}$ in the region $3300-3100 \mathrm{~cm}^{-1} \mathrm{of} \mathrm{the}$ free ligands disappeared in all the complexes indicating that the ligands are deprotonated in the complexes. The thioamide bands (IV) of the free ligands $\left(935-865 \mathrm{~cm}^{-1}\right)$ which have a major contribution from $v_{\mathrm{c}=\mathrm{s}}$ vibration is observed at $705 \pm 20 \mathrm{~cm}^{-1}$ in all the complexes, showing the coordination with deprotonated thiol sulphur. The coordination of $\mathrm{NH}_{3}$ in the complex $\left[\mathrm{ML}\left(\mathrm{NH}_{3}\right)\right]\left[\mathrm{M}=\mathrm{Ni}^{2+}\right.$ or $\mathrm{Zn}^{2+}, \mathrm{LH}_{2}=$ $\mathrm{H}_{2}$ Salmpth, $\mathrm{H}_{2}$ apmpth] is indicated by the appearance of bands at 3300-3100, 1590, 1280 and $850 \mathrm{~cm}^{-1}$ assigned to $v \mathrm{NH}_{3}, \delta \mathrm{NH}_{3}, \rho \mathrm{NH}_{3}$ sequentially. The pyridine ring breathing mode of vibration observed at $1025 \pm 5 \mathrm{~cm}^{-1}$ is regarded as a coordinated pyridine molecule. ${ }^{29}$ The $v_{\mathrm{C}=\mathrm{N}}$ for such complexes was observed at $1595 \pm 5 \mathrm{~cm}^{-1}$. In the far-infrared region, all the complexes displayed several bands at 460$410,400-380,360-320$ and $300-230 \mathrm{~cm}^{-1}$ those are assigned to $\mathrm{M}-\mathrm{O}, \mathrm{M}-\mathrm{N}, \mathrm{M}-\mathrm{S}, \mathrm{M}-\mathrm{Cl}$ str vibration, respectively.

It was observed that in slightly alkaline conditions $\mathrm{pH} \approx 7-8$ (or 9), the ligands $\mathrm{H}_{2}$ Salmpth and $\mathrm{H}_{2}$ apmpth, react with $\mathrm{Cr}(\mathrm{III}), \mathrm{Ni}(\mathrm{II})$ or $\mathrm{Zn}(\mathrm{II})$ salts giving rise to monoligated complexes $\left[\mathrm{CrL}\left(\mathrm{H}_{2} \mathrm{O}\right)_{2} \mathrm{Cl}\right]$, [ML(Py)] and $\left[\mathrm{ML}\left(\mathrm{NH}_{3}\right)\right],\left(\mathrm{M}=\mathrm{Ni}^{2+}\right.$ or $\left.\mathrm{Zn}^{2+}\right)$. It behaves in a dibasic tridentate manner and bonding takes place through $\mathrm{O}, \mathrm{N}$ and $\mathrm{S}$. In feebly alkaline or neutral conditions these and other ligands are found to act in a bidentate monobasic way bonding through azomethine $\mathrm{N}$-atom and $\mathrm{S}$-atom (via thioenolisation) keeping the $-\mathrm{OH}$ free.

\section{${ }^{1}$ H NMR Studies}

The signals due to $-\mathrm{NH}$ proton at $\delta \approx 11.8 \mathrm{ppm}$ and phenolic $-\mathrm{OH}$ at $\delta \approx 12.4-13.04 \mathrm{ppm}$ of ligands $\mathrm{H}_{2}$ Salmpth and $\mathrm{H}_{2}$ apmpth disappeared in its monoligated complexes of compositions $\left[\mathrm{CrL}\left(\mathrm{H}_{2} \mathrm{O}\right){ }_{2} \mathrm{Cl}\right]$, [ML(Py)] and [ML(NH3)], $\left(\mathrm{M}=\mathrm{Ni}^{2+}\right.$ or $\left.\mathrm{Zn}^{2+}\right)$, which shows the diabasic tridentate nature of these ligands in the complexes and bonding through $\mathrm{O}, \mathrm{N}$ and $\mathrm{S}$ atoms. In the neutral bischelates of $\mathrm{Ni}$ (II) and $\mathrm{Zn}$ (II) signals due to $-\mathrm{NH}$ proton at $\delta 11-12 \mathrm{ppm}$ of ligands disappears in complexes suggesting the thioenolization and coordination through deprotonated thiol sulphur atoms. In neutral, bischelates of $\mathrm{H}_{2}$ Salmpth and $\mathrm{H}_{2}$ apmpth of $\mathrm{Ni}(\mathrm{II})$ and $\mathrm{Zn}$ (II) the signals due to phenolic $-\mathrm{OH}$ at $\approx 12.4-13.04 \mathrm{ppm}$ remain unaffected in complexes and its downfield shift than normal phenolic - $\mathrm{OH}$ shows $\mathrm{H}$-bonding. The chemical shift $\delta$ 7.1-7.3 ppm of aromatic protons undergoes a slight downfield shift $\delta$ 7.4-7.9 ppm on complexation.

Thus, based on the above studies, the following tentative structures (Fig. 1.) have been proposed for the synthesized complexes.

\section{Antifungal Evaluation}

4-Methylpiperidine-1-carbothiohydrazide (4), $\quad N^{\prime}$-substituted-4-methylpiperidine-1-carbothiohydrazides (6a-f), monoligated pyridine complexes of $N^{\prime}$-substituted-4-methylpiperidine-1-carbothiohydrazides derived from salicylaldehyde $[\mathrm{M}(\mathrm{salmpth})(\mathrm{Py})]$ and bis-ligated complexes derived from furfural $\left[\mathrm{M}(\mathrm{fmpth})_{2}\right]$ and thiophene-2-aldehyde $\left[\mathrm{M}(\mathrm{talmpth})_{2}\right],\left(\mathrm{M}=\mathrm{Ni}^{2+}\right.$ or $\left.\mathrm{Zn}^{2+}\right)$ have been tested for antifungal activity. Antifungal activity of these compounds was evaluated by Agar-based disk-diffusion method ${ }^{30}$ against fungal strains $C$. albicans and $A$. niger using Griseofulvin as a standard drug. The results are 
shown in Table-4. All the tested compounds were found to possess moderate to significant antifungal activity. The complexes were found more active than ligands and the order of their activity was found as $[\mathrm{M}(\mathrm{salmpth})(\mathrm{Py})]>\left[\mathrm{M}(\mathrm{fmpth})_{2}\right]>\left[\mathrm{M}(\text { talmpth })_{2}\right]$.

The algacidal effect of $\mathrm{CrCl}_{3}$, the ligands- $\mathrm{H}_{2}$ salmpth, Hfmpth and Htalmpth and their chromium complexes were studied on the growth of green algae- chlorella. The ligands and complexes were found less toxic than metal chloride. The lesser algacidal effect of ligands and complexes concerning metal ions may be attributed to their larger size.

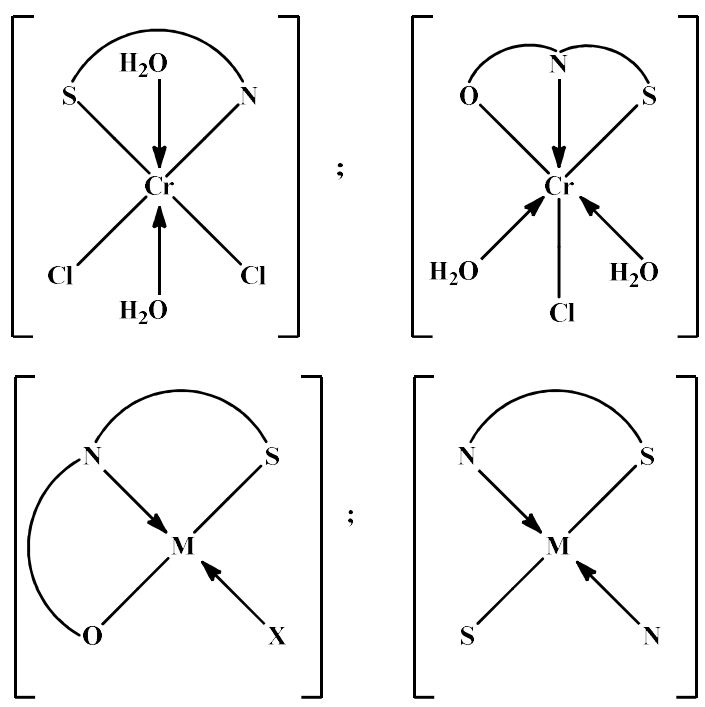

Fig. -1: Tentative Structures of the Complexes synthesized. Where $\mathrm{X}=\mathrm{Py}$ or $\mathrm{NH}_{3} ; \mathrm{M}=\mathrm{Ni}^{2+}$ or $\mathrm{Zn}^{2+}$. The parts involving $\mathrm{O}, \mathrm{N}$ and $\mathrm{S}$ atoms in the above structures represent the donor system of the ligands

Table-4: Antifungal Activity of 4-Methylpiperidine-1-carbothiohydrazide (4), $N^{\prime}$-Substituted-4-methylpiperidine-1carbothiohydrazides (6a-f) and Schiff base metal complexes (7w, 7y, 7i, 7j, 7k, 7l)

\begin{tabular}{c|c|c}
\hline Tested Compound & \multicolumn{2}{|c}{ Zone of Inhibition (in mm) } \\
\cline { 2 - 3 } at a Concentration of $100 \mu \mathrm{g} / \mathrm{ml}$ & C. albicans & A. niger \\
\hline Hmpth, 4 & 08 & 10 \\
\hline Hfmpth, 6a & 10 & 12 \\
\hline Htalmpth, 6b & 09 & 10 \\
\hline Hbenmpth, 6c & 05 & - \\
\hline $\mathrm{H}_{2}$ Salmpth, 6d & 12 & 10 \\
\hline$\left(\mathrm{H}_{2}\right.$ apmpth, 6e & 08 & 11 \\
\hline Hcycmpth, 6f & 12 & 10 \\
\hline$[\mathrm{Ni}($ Salmpth)Py], 7w & 17 & 19 \\
\hline$[\mathrm{Zn}($ Salmpth)Py], 7y & 19 & 20 \\
\hline$\left[\mathrm{Ni}(\mathrm{fmpth})_{2}\right], 7 \mathrm{i}$ & 14 & 11 \\
\hline$\left[\mathrm{Zn}(\mathrm{fmpth})_{2}\right], 7 \mathrm{j}$ & 15 & 14 \\
\hline$\left[\mathrm{Ni}(\text { talmpth })_{2}\right], 7 \mathrm{k}$ & 12 & 10 \\
\hline$\left[\mathrm{Zn}(\text { talmpth })_{2}\right], 71$ & 14 & 10 \\
\hline Griseofulvin & 22 & 25 \\
\hline
\end{tabular}

CONCLUSION

Three bidentate N, S donor type $N^{\prime}$-Substituted-4-methylpiperidine-1-carbothiohydrazides (6a-f) were synthesized using cyclohexanone, thiophene-2-aldehyde, benzaldehyde, salicylaldehyde, furfural and 2hydroxyacetophenone. These ligands were complexed with transition metals to produce their metal complexes. Physico-analytical data including spectral data and elemental analysis of ligands \& their metal(II) complexes were shown as per proposed structures. Potential ligands \& their metal complexes 
RASĀYAN J. Chem.

Vol. 14 | No. 3 |2068-2076| July - September | 2021

were tested for antifungal and algacidal potential. Results showed that metal complexes have greater antifungal activity in comparison to corresponding ligands. The greater antifungal activity of metal complexes was shown due to the chelation phenomenon, which coordinates the metal ion with ligands and makes a reduction in its polarity.

\section{ACKNOWLEDGEMENT}

Authors are indebted to Head, Department of Chemistry, Ranchi University, Ranchi-834002, Jharkhand, India for providing laboratory facilities.

\section{REFERENCES}

1. M. Li, H. Wu, S. Zhang, L. Sun, H. Ke, Q. Wei and S. Gao, European Journal of Inorganic Chemistry, 26, 811(2017), https://doi.org/10.1002/ejic.201601188

2. M. C. Heffern, V. Reichova, J.L. Coomes, A.S. Harney, E.A. Bajema and T.J. Meade, Inorganic Chemistry, 54, 9066(2015), https://doi.org/10.1021/acs.inorgchem.5b01415

3. X. M. Wang, S. Chen, R. Q. Fan, F. Q. Zhang and Y. L. Yang, Dalton Transactions, 44, 8107(2015), https://doi.org/10.1039/C5DT00057B

4. D. K. Maity and D. Ghoshal, Journal of the Indian Institute of Science, 97, 261(2017), https://doi.org/10.1007/s41745-017-0033-5

5. S. Ejaz, H. Nadeem, R.Z. Paracha, S. Sarwar and S. Ejaz, BMC Chemistry, 13, 115(2019), https://doi.org/10.1186/s13065-019-0631-6

6. E. Tas, M. Aslanoglu, A. Kilic, O. Kaplan and H. Temel, Journal of Chemical Research, 4, 242(2006), https://doi.org/10.3184/030823406776894292

7. A. Das, Y. Ren, C. Hessin and M. Desage-El Murr, Beilstein Journal of Organic Chemistry, 16, 858(2020), https://doi.org/10.3762/bjoc. 16.77

8. Y. Sano, N. Lau, A. C. Weitz, J. W. Ziller, M. P. Hendrich and A. S. Borovik, Inorganic Chemistry, 56, 14118(2017), https://doi.org/10.1021/acs.inorgchem.7b02230

9. A. Greatti, M. A. de Brito, A. J. Bortoluzzi and A. S. Ceccato, Journal of Molecular Structure, 688, 185(2004), https://doi.org/10.1016/j.molstruc.2003.10.008

10. H. Adams, S. Clunas and D. E. Fenton, Inorganic Chemistry Communications, 4, 667(2001), https://doi.org/10.1016/S1387-7003(01)00305-7

11. R. Ganguly, B. Sreenivasulu and J. J. Vittal, Coordination Chemistry Reviews, 252, 1027(2008), https://doi.org/10.1016/j.ccr.2008.01.005

12. M. Andruh, D. G. Branzea, R. Gheorghe and A.M. Madalan, CrystEngComm, 11, 2571(2009), https://doi.org/10.1039/B909476H

13. M. J. O'Donnell and R. L. Polt, Journal of Organic Chemistry, 47, 2663(1982), https://doi.org/10.1021/jo00134a030

14. H. Sachdeva, R. Saroj, S. Khaturia, D. Dwivedi and O.P. Chauhan, Journal of Chemistry, 2014, 1(2014), https://doi.org/10.1155/2014/848543

15. Y. Jia and J. Li, Chemical Reviews, 115, 1597(2015), https://doi.org/10.1021/cr400559g

16. C. W. Dikio, B.J. Okoli, F. M. Mtunzi, and G. Weaver, Cogent Chemistry, 3(1),1336864(2017), https://doi.org/10.1080/23312009.2017.1336864

17. K. L. Haas and K. J. Franz, Chemical Reviews, 109, 4921(2009), https://doi.org/10.1021/cr900134a

18. J. Devi, N. Batra and R. Malhotra, Spectrochimica acta. Part A, Molecular and Biomolecular Spectroscopy, 97, 397(2012), https://doi.org/10.1016/j.saa.2012.06.026

19. J. Mendham, R. C. Denney, J. D. Barnes and M. J. K. Thomas, Vogel's Textbook of Quantitative Chemical Analysis ed., Pearson Education Pvt. Ltd., New Delhi. Edition 6, Third Indian Reprint, pp. 454, 462, 472 (2003).

20. K. A. Jensen and J.F. Miquel, Acta Chemica Scandinavica, 189 (1970).

21. H. Gilman and A. H. Blatt, Organic Synthesis, Collective, ed. John Wiley, New York, Vol. I, pp. 448 (1966).

22. W. J. Geary, Coordination Chemistry Reviews, 7, 81(1971), https://doi.org/10.1016/S00108545(00)80009-0

23. K. Dey and D. Bandyopadhyay, Indian Journal of Chemistry, 31A, 34(1992)

24. B. N. Keshari and L. K. Mishra, Indian Journal of Chemistry, 20A, 883(1981)

2075

TRANSITION METAL COMPLEXES

Juhi Baranwal et al. 


\section{RASĀYAN J. Chem.}

Vol. 14 | No. 3 |2068-2076| July - September | 2021

25. N. N. Greenwood and A. Earnshaw, Chemistry of Elements, ed., Reed Elsevier India Pvt. Ltd., pp. 1029 (2005).

26. M. Kumar and T. Sharma, Journal of the Indian Chemical Society, 68, 539(1991).

27. A. Naik and V. K. Revankar, Journal of Chemical Sciences, 113, 285(2001).

28. B. B. Mahapatra, S. N. Dehury, S. Pratihari and S. N. Chaulia, Biokemistri, 24, 116(2012).

29. J. R. Dilworth and N. Wheatley, Coordination Chemistry Reviews, 199, 89(2000), https://doi.org/10.1016/S0010-8545(99)00185-X

30. E.I. Nweze, P. K. Mukherjee and M. A. Ghannoum, Journal of Clinical Microbiology, 48, 3750(2010), https://doi.org/10.1128/JCM.01357-10

[RJC-6204/2020] 